\title{
As Dimensões do Desenvolvimento: um estudo exploratório sob a perspectiva das ferramentas de avaliação
}

Hans Michael van Bellen ${ }^{1}$

\section{Resumo}

Este artigo procura explorar o conceito de desenvolvimento para em seguida abordar algumas ferramentas de avaliação que trabalham esse conceito a partir das diferentes dimensões. Em sua primeira parte, o artigo discute as diferentes dimensões do desenvolvimento para em seguida apresentar algumas ferramentas que trabalham com o conceito de desenvolvimento, sendo que tais ferramentas são analisadas a partir das diversas dimensões utilizadas no processo de avaliação. Observa-se que a dimensão econômica ainda é predominante nos sistemas de avaliação, entretanto, avanços significativos têm sido feitos no desenvolvimento $e$ na aplicação de sistemas de mensuração que utilizam a dimensão ambiental e social.

Palavras-chave: Desenvolvimento Sustentável. Indicadores. Avaliação.

\section{Introdução}

Os últimos anos foram palco de uma importante discussão acerca do significado do termo desenvolvimento e de qual a melhor maneira de medilo. Esse processo é, em parte, resultado das reflexões sobre a crise na relação sociedade e meio ambiente, que atinge proporções globais a partir da década de 1970. A tomada de consciência acerca dos problemas ambientais por parte da sociedade civil organizada, dos governos e até do mercado propiciou a realização de uma série de conferências que culminaram no conceito de desenvolvimento sustentável.

Esse conceito preconiza um tipo de desenvolvimento que garanta qualidade de vida para as gerações atuais e futuras sem a destruição da sua base

\footnotetext{
${ }^{1}$ Doutor pela Universidade Federal de Santa Catarina - UFSC. Departamento de Engenharia do Conhecimento. Campus Universitário Trindade. Caixa Postal 476. 88040-900. Florianópolis-SC-Brasil. E-mail: hansmichael@egc.ufsc.br.

Artigo recebido em: 17/08/2006. Aceito em: 10/09/2007. Membro do Corpo Editorial Científico responsável pelo processo editorial: Martinho Isnard Ribeiro de Almeida.
} 
de sustentação, que é o meio ambiente. O surgimento do conceito de desenvolvimento sustentável, que se tornou rapidamente uma unanimidade em todos os segmentos da sociedade, ocasionou o aprofundamento da discussão acerca do seu real significado teórico e prático.

Um dos resultados atuais desse aprofundamento é a avaliação do processo de desenvolvimento a partir de diferentes dimensões. As ideias do ecodesenvolvimento e o conceito de sustentabilidade acrescentaram ao conceito de desenvolvimento algumas dimensões que não eram normalmente observadas quando se pretendia avaliar este processo. Conhecer melhor as dimensões do desenvolvimento, juntamente com seus sistemas de avaliação, é o objetivo principal deste trabalho. Para alcançar esse objetivo foi utilizada uma abordagem qualitativa conduzida através de pesquisa documental. Foram utilizados diferentes documentos e fontes, que abordam o conceito de desenvolvimento observado a partir da ideia de sustentabilidade ao mesmo tempo em que algumas ferramentas de avaliação existentes foram analisadas. $\mathrm{O}$ artigo foi dividido em quatro partes: a primeira seção apresenta, de maneira resumida, os procedimentos metodológicos utilizados no trabalho. A seção seguinte explora as diferentes dimensões que, segundo alguns autores, estão de alguma maneira relacionadas à sustentabilidade para em seguida apresentar alguns sistemas de indicadores que trabalham com as dimensões do desenvolvimento utilizadas no artigo. A quarta e última procura destacar a importância de se observar o fenômeno do desenvolvimento a partir de diferentes dimensões e o papel destinado aos indicadores no processo de avaliação.

\section{Sustentabilidade e as Dimensões do Desenvolvimento}

Segundo Hardi e Barg (1997), embora seja possível apontar a direção do desenvolvimento para que ele seja "mais" sustentável, não é possível definir precisamente as condições de sustentabilidade de determinado desenvolvimento. O problema da definição, segundo esses autores, é de que não se pode capturar de maneira detalhada ou precisa a dinâmica da sustentabilidade humana e natural. A maior parte do debate contemporâneo sobre a sustentabilidade se refere a visões específicas de diferentes autores sobre aspectos distintos do conceito. Sem entrar nesse debate teórico, Hardi e Barg (1997) sugerem que as definições de sustentabilidade devem incorporar aspectos econômicos e ecológicos, juntamente com o bem-estar hu- 
mano. Tal desafio é reforçado por Moldan e Dahl (2007) que evidenciam, em um trabalho recente, os principais desafios conceituais relativos à mensuração da sustentabilidade. Esses autores destacam alguns aspectos críticos relacionados às dificuldades de mensuração do desenvolvimento, como a questão da diversidade, da relevância política das dimensões, das ideias de equidade e democracia, da mensurabilidade e da mensuração, dos dados disponíveis, entre outros.

Da mesma forma Rutherford (1997) afirma que um dos maiores desafios do desenvolvimento sustentável é a compatibilização da análise com a síntese. O desafio de construir um desenvolvimento dito sustentável, juntamente com indicadores que mostrem essa tendência, é o de compatibilizar o nível macro com o micro. No nível macro deve-se entender a situação do todo e sua direção de uma maneira mais geral e, dessa maneira, fornecer para o nível micro - em que se tomam as decisões - as informações importantes para as necessárias correções de rota. Rutherford (1997) coloca que a evolução da ecosfera é resultado da interação, inclusive humana, de milhares de decisões de nível micro. Por outro lado, existe uma interação do comportamento do micro em relação ao macro. É necessária uma abordagem integrada se o objetivo é compreender melhor o que seja um desenvolvimento ambientalmente sustentável e como ele pode ser avaliado. Além das esferas micro e macro, pode se discutir a própria integração das dimensões relacionadas ao processo de desenvolvimento. Para Morse et al. (2001), esse processo de juntar diferentes aspectos da realidade conduz, inevitavelmente, a julgamentos de valor e subjetividade.

Um dos princípios por trás de qualquer política que promova o desenvolvimento sustentável é que o desenvolvimento implica, em menor ou maior grau, alguma forma de degradação do meio ambiente (CAVALCANTI, 1997). O processo de globalização, que também se orienta em múltiplas perspectivas, aumentou ainda mais a pressão antrópica sobre o meio ambiente e até agora não tem sido capaz de responder a algumas das necessidades fundamentais do ser humano (STIGLITZ, 2002). Como vários autores mostram, existe um limite físico dentro do qual uma economia pode operar. Esse limite, para Daly (1994), é determinado pelo sistema maior dentro do qual uma economia deve funcionar: o sistema ecológico.

Para Rutherford (1997) deve-se olhar para o problema sob diferentes perspectivas. As principais esferas são, na opinião dele, a econômica, a ambiental e a social. Entretanto, não se deve, segundo ele, restringir essas 
diferentes esferas exclusivamente a seus domínios e sim ampliar os insights para o sistema como um todo. Também para Moldan e Dahl (2007; 1997), o conceito de sustentabilidade pode ser melhor entendido a partir de diversas dimensões, e estes autores, além de muitos outros (SAYER; CAMPBELL, 2004; DASGUPTA, 2004; MEINE, 2004), citam reiteradamente o caso das sociedades ocidentais cuja dimensão econômica tem sido predominantemente utilizada.

Talvez o fato de existirem diferentes concepções ambientalistas sobre a ideologia de desenvolvimento sustentável possa explicar a presença das diversas definições desse conceito. Entretanto, um conceito como o do desenvolvimento sustentável, com várias concepções, não pode ser operacionalizado, o que prejudica a implementação e a avaliação dos processos desse novo modelo de desenvolvimento. há uma necessidade em definir concretamente o conceito, verificando criticamente o seu significado e observando as diferentes dimensões que tal conceito abrange.

Nesse sentido, e considerando a sustentabilidade como um conceito dinâmico que engloba um processo de mudança, Sachs (1997) afirma que o conceito de desenvolvimento sustentável apresenta cinco dimensões principais: sustentabilidade social, sustentabilidade econômica, sustentabilidade ecológica, sustentabilidade geográfica e sustentabilidade cultural. Muito embora existam diversas sugestões, e controvérsias, acerca das dimensões que se relacionam com a sustentabilidade, é possível fazer uma análise inicial do conceito a partir dessas cinco dimensões.

\subsection{Sustentabilidade da Perspectiva Econômica}

Para Daly (1994; 1992), a teoria econômica deve atender três objetivos: alocação, distribuição e escala. Na economia, as questões relativas à alocação e à distribuição apresentam um tratamento consistente, tanto em termos teóricos quanto históricos. Entretanto, a questão referente à escala ainda não é formalmente reconhecida e não conta com instrumentos políticos de execução.

A alocação se refere à divisão relativa dos fluxos de recursos. Uma boa alocação é aquela que disponibiliza recursos em função das preferências individuais, em que tais preferências são avaliadas pela habilidade de pagar utilizando o instrumento do preço. A distribuição está relacionada à divisão dos recursos entre as pessoas. Já a escala se refere ao volume físico do fluxo 
de matéria e energia, de baixa entropia, retirada do ambiente em forma de matéria bruta e devolvida a esse meio como resíduos de alta entropia. A teoria econômica tem se abstraído da questão da escala de duas maneiras opostas: de um lado assume que o meio ambiente é uma fonte de recursos infinita e do outro lado que esse mesmo meio constitui depósito de resíduos de tamanho infinito em relação à escala do subsistema econômico. A crise surge quando a economia, ou o subsistema econômico, cresce de tal maneira que a demanda sobre o meio ambiente ultrapassa seus limites.

Nesse sentido, a sustentabilidade econômica abrange alocação e distribuição eficientes dos recursos naturais dentro de uma escala apropriada. O conceito de desenvolvimento sustentável, observado a partir da perspectiva econômica, segundo Rutherford (1997), vê o mundo em termos de estoques e fluxo de capital. Na verdade, essa visão não está restrita apenas ao convencional capital monetário ou econômico, mas está aberta a considerar capitais de diferentes tipos, incluindo o ambiental, ou natural, capital humano e capital social.

Para os economistas, o problema da sustentabilidade se refere à manutenção do capital em todas as suas formas. Rutherford (1997) afirma que muitos economistas ressaltam a semelhança entre a gestão de portfólios de investimento com a sustentabilidade, em que se procura maximizar o retorno mantendo o seu capital constante. Na gestão das carteiras é necessário muitas vezes mudar a proporção dos capitais investidos e o investimento também pode ser observado como uma estratégia para obter lucros futuros. Os economistas, ao contrário dos ambientalistas, tendem a ser otimistas em relação à capacidade humana de se adaptar a novas realidades ou circunstâncias e resolver problemas com sua capacidade técnica. No mundo econômico, para Rutherford (1997), o único elemento imprevisível é a raça humana, embora algumas linhas teóricas divirjam um pouco dessa abordagem afirmando que existe o interesse da manutenção do capital total e que variações dentro das diferentes categorias de capital podem ser compensadas por outro tipo de capital, o que remete à discussão sobre os graus de sustentabilidade de Pearce (TURNER et al.,1993).

Os economistas se aproximam das questões relativas à sociedade e meio ambiente pela discussão dos conceitos de sustentabilidade forte e fraca. Ambas estão baseadas no fato de que a humanidade deve preservar capital para as futuras gerações. O capital natural é constituído pela base de recursos naturais, renováveis e não renováveis, pela biodiversidade e a capacidade de absorção de dejetos dos ecossistemas. Dentro do conceito de sustentabilidade 
forte, todos os níveis de recursos devem ser mantidos e não reduzidos e no conceito de sustentabilidade fraca se admite a troca entre os diferentes tipos de capitais na medida em que se mantenha constante o seu estoque (TURNER et al., 1993).

Segundo Hardi e Barg (1997), essas duas abordagens partem da premissa de que o capital natural não deve ser tratado independentemente do sistema como um todo, mas sim como parte integrante dele. Na abordagem de MacNeill (MACNEILL et al., 1991), a integração entre ambiente e economia deve ser alcançada dentro do processo decisório, dentro dos diferentes setores como governo, indústria e ambiente doméstico, se o desejo é alcançar a sustentabilidade.

Em resposta às críticas constantes dos ambientalistas que afirmavam que os economistas utilizavam sistemas de contas incompletos e que desconsideravam, ou consideravam indevidamente, o capital natural, os economistas desenvolveram novos sistemas expandidos de contas para os sistemas nacionais. Alguns desses sistemas serão explorados posteriormente neste trabalho.

Também dentro da dimensão econômica, Bartelmus (1995) discute a sustentabilidade a partir da contabilidade e da responsabilidade. Para o autor, a contabilidade é pré-requisito para a gestão racional do meio ambiente e da economia, pois ele faz uma crítica aos meios convencionais de contabilidade na área financeira que procuram medir a riqueza de um país, e mostra os principais modelos que vêm sendo utilizados para ajuste das contas de um país. Os meios tradicionais para medir custo e capitais, os sistemas nacionais de contas, têm falhado por negligenciar, por um lado, a escassez provocada pela utilização de recursos naturais, que prejudica a produção sustentável da economia, e, por outro lado, a degradação da qualidade ambiental $e$ as consequências que essa degradação tem sobre a saúde $e$ o bem-estar humano. Adicione-se o fato de que gastos realizados para manutenção da qualidade ambiental são contabilizados como incremento nas receitas e produtos nacionais, sendo que tais despesas poderiam ser consideradas como custo de manutenção da sociedade. Para Bartelmus (1995), sistemas de contas integradas podem ser utilizados para avaliar dois aspectos da política econômica:

a) a sustentabilidade do crescimento econômico; e

b) a distorção estrutural da economia provocada pela produção e padrões de consumo doentios. 
A elaboração de políticas macroeconômicas deve reorientar o processo de desenvolvimento para um padrão mais sustentável através da internalização dos custos nos orçamentos de consumo doméstico e nos empreendimentos. Bartelmus (1995) ainda coloca a necessidade de suplantar os modelos tradicionais, que medem crescimento e performance da economia, por indicadores que incorporem a variável ambiental. Essa expansão do modelo de mensuração pode emitir os sinais de alarme necessários para reorientar a direção econômica rumo ao crescimento sustentável. O autor considera que uma análise mais detalhada da sustentabilidade, mesmo em relação à produção e ao consumo, naturalmente, deve considerar os fatores de capital humano e social, bem como seus efeitos sobre o progresso técnico, a substituição de bens e os serviços e desastres naturais.

Bartelmus (1995) revela que os mecanismos de comando e controle são ineficientes na proteção ambiental e na conservação de recursos naturais e que a aplicação de instrumentos de mercado pode ocorrer devido a taxas sobre efluentes emitidos, comércio de poluição, entre outras maneiras. Esses instrumentos procuram internalizar elementos externos da economia de modo a prover uma ótima alocação de recursos escassos. Sistemas de contabilidade integrada podem fornecer ajuda para esses instrumentos para medir o nível apropriado dos incentivos fiscais (subsídios) ou desincentivos (taxas).

Para Bartelmus (1995), a valoração monetária e econômica alcança seus limites quando se afasta dos resultados das atividades e processos humanos. A equidade, as aspirações culturais e a estabilidade política são elementos difíceis de quantificar, mesmo em termos físicos, e virtualmente impossíveis de reduzir em termos monetários, e, para esse autor, um conceito de desenvolvimento deve cobrir todos esses aspectos. O foco político da valoração monetária do crescimento econômico tem sido muito criticado pelos defensores de um tipo de desenvolvimento multiorientado. Existe uma crescente percepção de que é necessário considerar no planejamento, nas políticas e na ação em longo prazo, aspectos não monetários, demográficos, sociais e ambientais para realmente se alcançar a sustentabilidade.

Nesse sentido, Dahl (1997) também critica a linha teórica que advoga a manutenção do capital total e que considera o capital natural substituível pelo capital intelectual. Esse autor rechaça a utilização da monetarização pura e a criação e a utilização de indicadores únicos, argumentando que o mercado não atende a todas as necessidades humanas e sociais. Ele ainda faz um alerta sobre a importância das dimensões sociais no conceito de sustentabilidade e sobre a necessidade da utilização de indicadores relativos 
a aspectos sociais, como educação, sociedade civil e outros, quando se pretende avaliar o desenvolvimento sustentável.

Amartya Sen (2000) aborda indiretamente essa questão ao discutir os fins e os meios para o desenvolvimento. Para esse autor, a expansão das liberdades é o fim primordial e o principal meio do desenvolvimento e isso não se limita unicamente ao crescimento econômico. Kanbur (2002) trilha o mesmo caminho ao criticar as fraquezas da discussão puramente econômica do processo de desenvolvimento quanto tal discussão não contempla disciplinas como antropologia e sociologia.

\subsection{Sustentabilidade da Perspectiva Social}

Na sustentabilidade observada da perspectiva social, a ênfase é dada à presença do ser humano na ecosfera. A preocupação principal, dentro dessa linha, é a condição humana e os meios utilizados para aumentar a qualidade de vida. Rutherford (1997) argumenta, utilizando-se de um raciocínio econômico, que se deve preservar o capital social e humano e que o aumento desse montante de capital deve gerar dividendos.

Claramente, como já foi amplamente discutido, o conceito de bem-estar não é fácil de se construir nem de se medir. A questão da riqueza é importante, mas é apenas parte do quadro geral da sustentabilidade. Acesso a serviços básicos, água limpa e tratada, ar puro, serviços médicos, proteção, segurança e educação podem estar ou não relacionados com os rendimentos ou com a riqueza da sociedade. Para Sachs (1997), a sustentabilidade social refere-se a um processo de desenvolvimento que leve a um crescimento estável com distribuição equitativa de renda, gerando, com isso, a diminuição das atuais diferenças entre os diversos níveis na sociedade e a melhoria das condições de vida das populações.

\subsection{Sustentabilidade da Perspectiva Ambiental}

Para Rutherford (1997), na sustentabilidade da perspectiva ambiental, a principal preocupação é relativa aos impactos das atividades humanas sobre o meio ambiente. Essa preocupação é expressa pelo que os economistas chamam de capital natural. Nessa visão, a produção primária, oferecida pela natureza, é a base fundamental sobre a qual se assenta a espécie humana. 
Foram os ambientalistas, principais atores dessa abordagem, que desenvolveram o modelo denominado PSR (Pressure, State e Response) para indicadores ambientais e que o defendem para as outras esferas.

Sustentabilidade ecológica significa ampliar a capacidade do planeta através da utilização do potencial encontrado nos diversos ecossistemas, ao mesmo tempo em que se mantém um nível mínimo de deterioração desses ecossistemas. Deve-se reduzir a utilização de combustíveis fósseis, diminuir a emissão de substâncias poluentes, adotar políticas de conservação de energia e de recursos, substituir recursos não renováveis por renováveis e aumentar a eficiência em relação aos recursos utilizados (SACHS, 1997).

\subsection{Sustentabilidade da Perspectiva Geográfica}

A sustentabilidade geográfica pode ser alcançada por meio de uma melhor distribuição dos assentamentos humanos e das atividades econômicas. Trata-se, como afirma Guimarães (1997), de incluir no discurso do desenvolvimento os impactos do crescimento demográfico e de sua distribuição. Como afirma esse autor, é preciso adequar as tendências esperadas ou projetadas de distribuição espacial da população e seus processos de migração com as expectativas de crescimento regional. Ao mesmo tempo, é essencial procurar uma configuração rural-urbana mais adequada para proteger a diversidade biológica e melhorar a qualidade de vida das pessoas.

\subsection{Sustentabilidade da Perspectiva Cultural}

Por último, a sustentabilidade cultural, a mais difícil de ser concretizada, segundo Sachs (1997), está relacionada ao caminho da modernização sem o rompimento da identidade cultural dentro de contextos espaciais específicos. Para Sachs (1997), o conceito de desenvolvimento sustentável refere-se a uma nova concepção dos limites e ao reconhecimento das fragilidades do planeta, ao mesmo tempo em que enfoca o problema socioeconômico e a satisfação das necessidades básicas das populações.

A discussão anterior revela a existência de uma variedade de aspectos relacionados às diferentes dimensões da sustentabilidade. Muito embora o ponto de partida das diversas abordagens seja distinto, existe um reconhecimento de que há um espaço de interconexão ou interseção entre esses dife- 
rentes campos. Ao mesmo tempo, alguns autores destacam outras dimensões da sustentabilidade. A utilização seletiva dessas dimensões, por parte de diferentes autores, pode estar relacionada aos diferentes campos do ambientalismo. Uma interpretação desses campos é dada por Pearce que descreve diferentes ideologias associadas ao movimento ambiental (TURNER et al., 1993). Esses campos ideológicos são apresentados no Quadro 1.

Independentemente de tais campos, as dimensões do desenvolvimento têm sido utilizadas na construção de sistemas de indicadores relacionados à sustentabilidade. A próxima seção aborda alguns desses sistemas de indicadores de sustentabilidade, considerando como ponto de partida essas dimensões do conceito de desenvolvimento sustentável.

\begin{tabular}{|c|c|c|c|c|}
\hline Cornucopiana & Adaptativa & Comunalista & Ecologia Profunda & \\
\hline $\begin{array}{l}\text { Exploração de } \\
\text { recursos, orientação } \\
\text { pelo crescimento }\end{array}$ & $\begin{array}{l}\text { Conservacionismo } \\
\text { de recursos, posição } \\
\text { gerencial }\end{array}$ & $\begin{array}{l}\text { Preservacionismo } \\
\text { de recursos }\end{array}$ & $\begin{array}{l}\text { Preservacionismo } \\
\text { profundo }\end{array}$ & $\begin{array}{c}\text { RÓTULO } \\
\text { AMBIENTAL }\end{array}$ \\
\hline $\begin{array}{l}\text { Economia } \\
\text { antiverde, livre } \\
\text { mercado }\end{array}$ & $\begin{array}{l}\text { Economia verde, } \\
\text { mercado verde } \\
\text { conduzido por } \\
\text { instrumentos de } \\
\text { incentivos } \\
\text { econômicos }\end{array}$ & $\begin{array}{l}\text { Economia verde } \\
\text { profunda. Economia } \\
\text { steady-state, } \\
\text { regulação } \\
\text { macroambiental }\end{array}$ & $\begin{array}{l}\text { Economia verde } \\
\text { muito profunda, } \\
\text { forte regulação para } \\
\text { minimizar a tomada } \\
\text { de recursos }\end{array}$ & TIPO DE ECONOMIA \\
\hline $\begin{array}{c}\text { Objetivo } \\
\text { econômico, } \\
\text { maximização do } \\
\text { crescimento } \\
\text { econômico. } \\
\text { Considera que o } \\
\text { mercado livre em } \\
\text { conjunção com o } \\
\text { progresso técnico } \\
\text { deve possibilitar a } \\
\text { eliminação das } \\
\text { restrições relativas } \\
\text { aos limites e à } \\
\text { escassez }\end{array}$ & $\begin{array}{c}\text { Modificação do } \\
\text { crescimento } \\
\text { econômico, norma } \\
\text { do capital constante, } \\
\text { alguma mudança de } \\
\text { escala }\end{array}$ & $\begin{array}{c}\text { Crescimento } \\
\text { econômico nulo, } \\
\text { crescimento } \\
\text { populacional nulo. } \\
\text { Perspectiva } \\
\text { sistêmica, saúde do } \\
\text { todo (ecossistema), } \\
\text { hipótese de Gaia e } \\
\text { suas implicações }\end{array}$ & $\begin{array}{l}\text { Reduzida escala da } \\
\text { economia e da } \\
\text { população. } \\
\text { Imperativa mudança } \\
\text { de escala, } \\
\text { interpretação literal } \\
\text { de Gaia }\end{array}$ & $\begin{array}{l}\text { ESTRATÉGIA DE } \\
\text { GESTÃO }\end{array}$ \\
\hline $\begin{array}{l}\text { reitos e interesses } \\
\text { dos indivíduos } \\
\text { contemporâneos, } \\
\text { valor instrumental } \\
\text { na natureza }\end{array}$ & $\begin{array}{c}\text { Eqüidade intra e } \\
\text { intergeracional } \\
\quad \text { (pobres } \\
\text { contemporâneos e } \\
\text { gerações futuras), } \\
\text { valor instrumental } \\
\text { na natureza }\end{array}$ & $\begin{array}{l}\text { Interesse coletivo } \\
\text { sobrepuja o } \\
\text { interesse individual, } \\
\text { valor primário dos } \\
\text { ecossistemas e valor } \\
\text { secundário para } \\
\text { suas funções e } \\
\text { serviços }\end{array}$ & $\begin{array}{l}\text { Bioética (direitos e } \\
\text { interesses } \\
\text { conferidos a todas } \\
\text { as espécies), valor } \\
\text { intrínseco da } \\
\text { natureza }\end{array}$ & ÉTICA \\
\hline $\begin{array}{c}\text { Sustentabilidade } \\
\text { Muito Fraca }\end{array}$ & $\begin{array}{c}\text { Sustentabilidade } \\
\text { Fraca }\end{array}$ & $\begin{array}{c}\text { Sustentabilidade } \\
\text { Forte }\end{array}$ & $\begin{array}{l}\text { Sustentabilid ade } \\
\text { Muito Forte }\end{array}$ & $\begin{array}{c}\text { GRAU DE } \\
\text { SUSTENTABILIDADE }\end{array}$ \\
\hline
\end{tabular}

Quadro 1: Dimensões do ambientalismo

Fonte: Adaptado de Turner et al. (1993) 


\section{Ferramentas de Avaliação e as Dimensões do Desenvolvimento}

Existem alguns tipos de sistemas de avaliação para mensurar o grau de sustentabilidade do desenvolvimento, mas, conforme descrito anteriormente, o próprio conceito de desenvolvimento abrange muitas questões e dimensões diferentes. Isso se reflete nos sistemas de indicadores que estão sendo desenvolvidos e utilizados para esse fim. Esta seção aborda alguns desses sistemas de indicadores que atuam em diferentes dimensões do conceito de desenvolvimento procurando mensurar o seu grau de sustentabilidade.

Quando se trata de indicadores ambientais algumas aproximações foram feitas utilizando o sistema de média (água, ar, solo, recursos), ou o sistema de metas, utilizando os parâmetros legais como objetivos dos indicadores. Entretanto, atualmente, a maior fonte de indicadores ambientais é a publicação regular da OECD - Organization for Economical Cooperation and Development (1993) que fornece um primeiro mecanismo para monitoramento do progresso ambiental para os países que fazem parte dessa instituição. O grupo de indicadores dessa organização é limitado em tamanho, mas cobre uma vasta área de questões ambientais, representando um grupo comum de indicadores dos países-membros e, adicionalmente, incorpora indicadores derivados de alguns grupos setoriais e de sistemas de contabilidade ambiental.

O sistema utiliza-se do modelo PSR (Pressure-State-Response) que é um dos sistemas que vem adquirindo cada vez mais importância internacional. Esse sistema foi desenvolvido a partir do sistema Stress-Response que é aplicado em ecossistemas para a primeira classificação dos indicadores. $\mathrm{O}$ sistema PSR assume implicitamente que existe uma causalidade na interação dos diferentes elementos da metodologia.

Os indicadores de pressão ambiental (P) representam ou descrevem pressões das atividades humanas exercidas sobre o meio ambiente, incluindo os recursos naturais. Os indicadores de estado ou condição (S) e se referem à qualidade do ambiente e à qualidade e quantidade de recursos naturais. Dessa maneira, eles refletem o objetivo final da política ambiental. Indicadores da condição ambiental são projetados para dar uma visão geral da situação do meio ambiente e seu desenvolvimento no tempo. 
Indicadores de resposta $(\mathrm{R})$ mostram a extensão e a intensidade das reações da sociedade em responder às mudanças e às preocupações ambientais. As mudanças e as preocupações se referem à atividade individual e coletiva para mitigar, adaptar ou prevenir os impactos negativos, induzidos pelo homem, sobre o meio ambiente, para interromper ou reverter danos ambientais já infligidos e para preservar e conservar a natureza e os recursos naturais.

Os objetivos principais do trabalho da OECD são:

a) rastreamento do progresso ambiental (monitoramento do ambiente e de suas mudanças no tempo);

b) integração entre preocupações ambientais e políticas públicas; $e$

c) integração entre preocupações ambientais e política econômica.

Uma abordagem da dimensão ecológica faz referência a indicadores relacionados a transporte e fluxo de material, Total Material Consumption (TMC) e a recursos e energia, Total Material Input (TMI). Nesse caso, muito embora o propósito da ferramenta seja ambiental, a metodologia utilizada para cálculo é econômica. A vantagem do TMI e do TMC é que esses indicadores fornecem uma ligação entre o consumo de materiais e seus impactos na natureza. Outro aspecto interessante está ligado à chamada desmaterialização do consumo, com estudos na Alemanha, Áustria e França (WEIZSÄCKER et al., 1995).

O fluxo de materiais e energia é um importante - mas não único - aspecto referente à sustentabilidade, é ainda um dos mais consideráveis quando se deseja manter o capital natural, trata-se da manutenção da diversidade biológica. Isso não é importante apenas no sentido de que seu potencial oferece soluções para benefício humano que ainda não são conhecidas, mas também porque a diversidade biológica fornece a base de estabilidade para o sistema no qual os seres humanos vivem. Nesse campo, outro indicador parcialmente conhecido é o Biodiversity Indicators for Policy-Makers do World Resources Institute (WRI), (HAMMOND et al., 1995). Esse sistema constitui-se de 22 indicadores, fornecendo informações úteis para os níveis nacional e internacional. Embora exista um grande número de dados disponíveis, algumas deficiências ainda podem ser notadas. Esses dados devem ser complementados com dados de gestão e economia, para auxiliar na determinação de prioridades e na tomada de decisões específicas para proteção ambiental. 
Quanto à dimensão econômica, sistemas de indicadores relacionados ao desenvolvimento sustentável têm surgido com mais força nos últimos tempos. No sentido de abordar a questão ambiental nos sistemas de mensuração econômica, a divisão de estatística da Organização das Nações Unidas, UNSD (United Nations Statistics Division) desenvolveu um sistema "paralelo" para integrar mais do que modificar o sistema atualmente utilizado. Com isso, visando a uma experimentação mais abrangente, a UNSD lançou uma versão de seu modelo no manual de Contabilidade Integrada Ambiental e Econômica (SEEA - System of Integrated Environmental and Economic Accounting, UNITED NATIONS, 1993). De acordo com alguns estudos, um sistema de contas "verdes" não só é realizável, mas também pode fornecer - mesmo que inicialmente e de maneira indicativa apenas - informações valiosas em termos de desenvolvimento de políticas e planejamento.

O propósito fundamental do sistema de contas integradas é o de cobrir a deficiência dos sistemas tradicionais de contas. Os objetivos principais das diferentes versões do sistema são, segundo Bartelmus (1995):

a) Segregação e elaboração de todos os fluxos e estoques relativos ao meio ambiente em relação ao sistema tradicional: o objetivo principal é colocar separadamente os investimentos em proteção ambiental. Essas despesas têm sido consideradas parte do custo necessário para compensar os impactos negativos do crescimento econômico.

b) Ligação da contabilidade física com a contabilidade ambiental monetária dentro de balanços: propriedade física compreende o estoque total de reservas de recursos e muda constantemente; os pioneiros nessa área têm sido a Noruega e a França, com sua contabilidade de patrimônio natural, e, mais recentemente, pelos estatísticos alemães com a contabilidade de fluxo de matéria e de energia.

c) Avaliação ambiental de custo-benefício: o sistema SEEA amplia e complementa o sistema tradicional, pois considera os custos (a utilização - depleção - dos recursos naturais na produção e na demanda final e as mudanças na qualidade ambiental - degradação) resultantes da poluição e outros impactos da produção, consumo e eventos naturais, por um lado, e os benefícios ou proteção e melhoria ambiental, por outro. 
d) Contabilidade para manutenção de riqueza factível: o sistema amplia o conceito de capital incorporando não apenas o capital gerado pela produção humana, mas também o capital natural. O capital natural inclui recursos naturais não renováveis como terra, solo e subsolo, e recursos cíclicos como ar e água. O processo de formação de capital é ampliado dentro do conceito de acumulação de capital.

e) Elaboração e mensuração de indicadores de estoques e receitas ajustados ao meio ambiente: a consideração dos custos da depleção dos recursos naturais e as mudanças na qualidade ambiental permitem o cálculo de agregados macroeconômicos modificados. O resultado é o Produto Interno Líquido Ambientalmente Ajustado (EDP - Environmentally Adjusted net Domestic Product).

Outro sistema interessante de indicadores econômicos é o MEP, Monitoring Environmental Progress, desenvolvido pelo Banco Mundial (WORLD BANK, 1995). O sistema se fundamenta na ideia de que a sustentabilidade é medida por uma riqueza per capita não decrescente. Os primeiros relatórios de riqueza foram produzidos pelo MEP em 1995 (O`CONNOR, 1997). O MEP amplia o conceito de contabilidade ambiental incorporando ao balanço os recursos humanos (investimentos em educação, treinamento, saúde) e infraestrutura social (associações). Apesar de suas limitações (avaliabilidade e confiabilidade de dados), esse sistema traz algumas importantes informações aos tomadores de decisão. A produção de bens, vista como principal determinante de riqueza em vários países, expressa efetivamente apenas um quinto da riqueza real na maioria dos países, pobres ou ricos. A análise de riqueza considera que o mix de bens possa mudar com o tempo, embora algumas fronteiras críticas devam ser respeitadas dentro de cada categoria.

Esse mix é influenciado pelo fluxo de receitas, produção e despesas. O MEP enfatiza que o meio para se criar riqueza é o fluxo de poupança verdadeiro, calculado a partir do resultado da produção ou receita menos o consumo, a depreciação dos bens de manufatura e a redução de recursos naturais. Alguns trabalhos mostram que a curva de Produto Interno Bruto - PIB acompanha a curva do GNI (Green net National Income) com exceção de alguns poucos países. Esse sistema apresenta aspectos positivos na medida em que mostra que muitas vezes não existe produção de riqueza e, sim, apenas, substituição de bens. Na segunda edição do MEP, as medidas foram 
refinadas tendo como base o conceito de riqueza como a soma de quatro componentes ou quatro tipos de capital: produzido, natural, humano e social.

A maior tentativa de ajuste das contas econômicas convencionais nos anos recentes tem sido o ISEW (Index of Sustainable Economic Welfare) que foi desenvolvido inicialmente por Daly e Cobb (1989). O ISEW ajusta as contas tradicionais com subtrações de influências negativas (referentes, por exemplo, à depleção de recursos naturais, desigualdade econômica e danos ambientais) e adições de influências positivas como o trabalho doméstico. O trabalho inicial do ISEW foi revisto por Cobb, em 1994, e forma agora a base do GPI (Genuine Progress Indicator).

O sistema ISEW fornece uma nova visão da mudança do bem-estar econômico no tempo. Esse sistema toma como base a medida do gasto do consumidor, que está relacionado ao Produto Interno Bruto, e então faz ajustes para 18 aspectos econômicos da vida cotidiana que o PIB tradicional ignora. As principais diferenças entre o PIB e o ISEW são:

a) as despesas com custos sociais e ambientais são retiradas (investimentos defensivos);

b) o dano ambiental em longo prazo e a depreciação do capital natural são considerados;

c) a formação de capital de manufatura humano é incluída;

d) mudanças na distribuição de receitas são incluídas (incremento de receita tem peso desigual dependendo da classe social do indivíduo); e

e) o valor do trabalho caseiro é incluído.

Existem também numerosos exemplos de indicadores relacionados à dimensão social da sustentabilidade. Um dos que tem merecido maior destaque ultimamente é o Índice de Desenvolvimento Humano (HDI - Human Development Index). Esse índice foi desenvolvido através do Programa das Nações Unidas para o Desenvolvimento que, em seu relatório, Human Development Report (UNDP, 1990; 1995), sugere que a medida do desenvolvimento humano deve focar três elementos principais: longevidade, conhecimento e padrão de vida decente.

Para o item longevidade o padrão considerado é a expectativa de vida no nascimento. A importância da expectativa de vida é sugerida pela crença de que uma vida longa contém uma valoração positiva por si só, e está agre- 
gada a outros benefícios indiretos (como uma adequada nutrição e um bom sistema de saúde). Tais associações fazem da expectativa de vida um importante indicador do desenvolvimento humano, especialmente em virtude muitas vezes da falta de dados existente em relação aos níveis de alimentação e saúde.

O conhecimento se refere à capacidade de leitura ou grau de alfabetização que reflete apenas grosseiramente o acesso à educação - particularmente uma educação de qualidade - que é necessária para a vida produtiva dentro da sociedade moderna. O grau de leitura é o primeiro passo no processo de aprendizagem na construção do conhecimento, sendo assim esse indicador é importante em qualquer medida do desenvolvimento humano. Embora outros indicadores tenham que ser trabalhados para se perceber efetivamente o grau de conhecimento dentro de determinado grupo, o grau de "literacy" tem uma clara importância na investigação do Índice do Desenvolvimento Humano.

O padrão de vida decente é, segundo Moldan e Bilharz (1997), a medida mais difícil de obter simplesmente. Essa dificuldade está relacionada com a necessidade de dados confiáveis e específicos, mas devido à escassez dessa variedade de dados precisa-se, para início, utilizar o melhor dos indicadores de receita. $\mathrm{O}$ indicador mais confiável e com maior facilidade de obtenção é a receita per capita, mas a existência de bens e serviços que não são de mercado e as distorções existentes dentro dos sistemas de contas nacionais, os quais já foram discutidos anteriormente, tornam esse sistema não muito adequado para comparações. Esses dados, porém, podem ser aperfeiçoados utilizando-se ajustes dentro do Produto Interno Bruto, que poderiam melhorar a aproximação em relação à capacidade efetiva de compra de bens e de comando sobre os recursos necessários para se viver dentro de um padrão adequado.

Outra abordagem da sustentabilidade dentro da esfera social é o conceito de privação humana. A discussão mais comum sobre sustentabilidade afirma que a geração atual deve deixar para as gerações vindouras no mínimo uma riqueza igual (incluídos capital humano, físico, natural e social) à existente nos dias atuais. A sustentabilidade é um conceito fundamentalmente normativo, ela implica a manutenção, para cada geração, de um nível socialmente aceitável de desenvolvimento humano. A questão que se coloca é a de qual o padrão mínimo aceitável para uma vida que "valha a pena" (ANAND; SEN, 1994). A resposta deve ser não um estoque cumulativo de 
riqueza, mas sim um nível particular e adequado de desenvolvimento humano, e o conceito que mais se ajusta ao nível de desenvolvimento humano é a ausência de privação. Esse padrão mínimo define as obrigações da sociedade para com cada um dos seus membros, fornecendo a eles pelo menos o mínimo necessário para subsidiar seu próprio desenvolvimento como seres humanos, livres de necessidades e de privação.

Uma sustentabilidade do tipo normativa não pode ser avaliada adequadamente em termos monetários. A avaliação envolve necessariamente o estabelecimento de padrões ou metas não monetárias. Alguns advogam que o não declínio do Índice de Desenvolvimento Humano (HDI) poderia ser tomado como medida de sustentabilidade normativa, da mesma maneira que o não declínio da riqueza total produtiva pode ser uma medida técnica da sustentabilidade. Mas a maioria das variáveis do HDI reflete a condição média de uma dada população e dessa maneira não pode mostrar que uma parcela significante dessa mesma população pode não ter os requisitos básicos de uma sobrevivência digna atendidos.

Embora semelhante ao HDI, o CPM (Capability Poverty Measure) é mais adequado para monitorar o nível de privação humana (MCKINLEY, 1997). O desenvolvimento humano é definido pela expansão das capacidades e a privação pela ausência de capacidades básicas ou essenciais. Capacidades são fins e elas se refletem na qualidade de vida das pessoas. O CPM difere do HDI quanto ao foco no ser humano pela ausência de capacidades, mais do que o nível médio de capacidades. Para evitar a confusão entre fins e meios, esse índice não utiliza as receitas como indicador de desenvolvimento humano. O CPM é um índice composto que utiliza a média aritmética de três indicadores:

a) a percentagem de crianças com menos de cinco anos com subnutrição (peso abaixo do normal);

b) a percentagem de mulheres com 15 anos ou mais que são analfabetas; $e$

c) a percentagem de nascimentos que não são atendidos por pessoas treinadas da área da saúde.

Essas variáveis cobrem uma ampla área: indicadores de saúde e nutrição para a população como um todo, acesso a serviços de saúde e indicadores básicos de educação. Um aspecto importante do método refere-se ao fato 
de que as variáveis sejam escolhidas para detectar diretamente a ausência de necessidades humanas básicas e, no caso do método CPM, essas necessidades são:

a) uma vida saudável e bem nutrida;

b) seres humanos alfabetizados e com capacidade de discernimento; $e$

c) seres humanos capazes de uma reprodução sadia e segura.

Existem casos em que não é possível determinar indicadores adequados que reflitam diretamente essa privação. Como substituto, os indicadores de acesso podem ser utilizados, como acesso à água potável, a instalações sanitárias adequadas e a serviços médicos. A diferença desse método, novamente, é o foco no ser humano. Diferentemente dos outros métodos baseados nas necessidades humanas e que procuram medir o acesso a tais necessidades dentro de um grupo, o CPM realça a privação, ou o não acesso a elementos essenciais para um padrão de vida digno (MCKINLEY, 1997).

Outro sistema que apesar de pouco desenvolvido tem chamado a atenção recentemente é o relacionado ao conceito de Capital Social. A maioria dos sistemas de indicadores sociais está relacionada ao que foi chamado por Sen (1987) de capacidades e liberdades. O Índice de Desenvolvimento Humano (HDI) é um bom exemplo de um grupo de componentes, procurando medir um padrão de vida livre e sem privação, muito embora exista muito espaço para melhorias. Para países com um nível de desenvolvimento relativamente baixo, o CPM é certamente mais prioritário. Mas MacGillivray (1997) aborda uma questão importante: uma vez que os pré-requisitos para o desenvolvimento humano sejam atendidos (alfabetização, saúde, conhecimento, receitas adequadas) como se pode realmente afirmar que as pessoas utilizarão esse potencial para exercer uma vida plena de significados dentro da sociedade?

Para esse autor, a participação dentro da sociedade é mais do que a inexistência de obstáculos para se alcançar alguns objetivos. Ele afirma que existem meios de se definir o desenvolvimento social não individual, pois utiliza o conceito de capital social para isso, conceito que, segundo Coleman (1988), representa a habilidade das pessoas de trabalharem juntas para um fim comum em grupos ou dentro das organizações. Putman (1994) descreve o capital social como uma característica da organização social, como as redes, as normas que facilitam a coordenação e cooperação em benefício mú- 
tuo. Tais associações fornecem a base de cooperação dentro da sociedade e o capital social pode ser descrito como a participação no processo decisório ou integração social.

As bases teóricas e empíricas sobre o capital social ainda estão em sua fase inicial. Existem algumas pesquisas pioneiras que mostram que a participação em corais, ou agremiações de esporte e cooperativas é um importante indicador de uma efetiva democracia local. Os resultados de um estudo recente do Banco Mundial mostram como o capital social pode trazer um significativo benefício para o bem-estar doméstico e MacGillivray (1997) mostra alguns estudos apontando a correlação entre o capital social e o aumento do bem-estar local.

Entretanto, a dificuldade de fornecer e desenvolver indicadores para os aspectos humanos do desenvolvimento sustentável persiste. Dentro de uma observação mais detalhada existe uma mistura entre o bem-estar dos indivíduos (como os aspectos relativos à saúde, educação, ausência de pobreza) e questões relacionadas ao capital social, que está longe de ter uma definição universal, incluindo aspectos como o papel das leis, a estabilidade, a confiança, as redes sociais, o acesso a informações, as instituições adequadas e a ausência de corrupção. Esses modelos vêm sendo amplamente reconhecidos como elementos críticos para a transição rumo a um tipo de sociedade mais sustentável (UNITED NATIONS, 1997). Esses indicadores são difíceis de serem capturados em um ou alguns poucos indicadores quantitativos. Parece ser mais fácil caracterizar tais aspectos em termos de padrões mais qualitativos do que em números apenas.

Se o objetivo é aplicar métodos numéricos não existe praticamente nenhum material avaliável na área de capital social. Ao mesmo tempo, há algumas metodologias bem estabelecidas na área de capital humano. Alguns exemplos são mortalidade infantil, expectativa de vida, as medidas de pobreza da Comissão sobre Desenvolvimento Sustentável da ONU (CSD - UNITED NATIONS, 1996a) e o Índice de Desenvolvimento Humano da UNDP (United Nations Development Program, 1995).

Para as perspectivas de desenvolvimento sustentável na área de capital social, deve-se incluir a questão de como este último deve ser mensurado, de uma maneira equilibrada, com o capital humano e o capital natural (SERAGELDIN, 1996; SERAGELDIN; STEER 1994). Outra questão importante que deve ser observada é a ausência de indicadores não triviais na dimensão institucional do desenvolvimento sustentável. Esse aspecto pode 
ser considerado atualmente um dos maiores problemas nos projetos relativos a indicadores de sustentabilidade.

Como se observa, existem várias tentativas, dentro das diferentes dimensões, de procurar avaliar a sustentabilidade. isso ocorre apesar das lacunas teóricas e empíricas que existem nesses modelos unidimensionais e da quase ausência de projetos de indicadores relacionados a determinadas dimensões, como é o caso da dimensão institucional. Entretanto, a partir desses sistemas mais específicos, alguns sistemas que procuram integrar as diversas dimensões da sustentabilidade foram elaborados. O DSR é um dos métodos mais conhecidos dentre os que procuram integrar as diversas dimensões do desenvolvimento sustentável. O método de avaliação Driving Force - State - Response (DSR) foi adotado pela Comissão de Desenvolvimento Sustentável das Nações Unidas em 1995 como uma ferramenta capaz de organizar informações sobre o desenvolvimento. O objetivo do programa é tornar acessíveis aos tomadores de decisão os indicadores relacionados ao desenvolvimento sustentável, no nível nacional, definindo-os, elucidando as suas metodologias e fornecendo treinamento e capacitação.

Nesse sistema, o item Driving Force representa as atividades humanas, processos e padrões que causam impacto no desenvolvimento sustentável. Esses indicadores fornecem uma medida das causas das mudanças, negativas ou positivas, no estado de desenvolvimento sustentável. Exemplos são a taxa de crescimento da população e a taxa de emissão de $\mathrm{CO} 2$.

Os indicadores do item State fornecem uma medida do estado do desenvolvimento sustentável, ou um aspecto particular dele, em um determinado momento. A esse item pertencem indicadores qualitativos e quantitativos como número estimado da população na escola, indicador de estado do nível educacional ou a concentração de poluentes no ambiente, que é uma medida da qualidade do ar nas áreas urbanas.

Indicadores do item Response mostram as opções políticas e outras respostas para as mudanças no estado do desenvolvimento sustentável. Esses indicadores fornecem uma medida da disposição e efetividade da sociedade em fornecer respostas. Algumas respostas para mudar o estado em relação ao desenvolvimento sustentável podem ser a legislação, regulação, instrumentos econômicos e atividades de informação, etc. Exemplos de indicadores do tipo Response incluem tratamento de água poluída e gastos na diminuição da poluição. 
Todos os capítulos da Agenda 21 estão refletidos nesse sistema dentro do qual estão contidas quatro dimensões do desenvolvimento sustentável: social, econômica, ambiental e institucional. Assume-se que o desenvolvimento sustentável inclui componentes dessas quatro categorias que estão inter-relacionados (UNITED NATIONS, 1996a).

O sistema DSR foi desenvolvido basicamente a partir do sistema PSR utilizado pela OECD em seus trabalhos sobre indicadores ambientais. No sistema DSR, o item Pressure (P) foi substituído por Driving-Force (D) para que fosse possível incorporar os aspectos sociais, econômicos e institucionais do desenvolvimento sustentável. Existem outras metodologias que utilizam algumas variações do sistema DSR, fazendo algumas alterações nele. Um exemplo é a subdivisão da categoria State $(S)$ em outras duas categorias como no caso do sistema PSIR (Pressure-State-Impact-Response) utilizado pela UNEP. Vários autores consideram que, em alguns aspectos, essa divisão pode trazer insights valiosos na ordenação de políticas públicas, mas por outro lado não atende a um dos critérios principais que seria o de simplificar tais indicadores ao máximo para os tomadores de decisão.

O sistema DSR pode ser utilizado também para avaliações setoriais e a indústria desempenha um importante papel no contexto do desenvolvimento sustentável em pelo menos dois aspectos: a produção industrial é uma das fontes geradoras de problemas ambientais e, em contrapartida, representa um componente importante em termos tecnológicos e econômicos na busca de soluções para a sustentabilidade. Esses dois aspectos estão ligados aos itens Driving Force e State do método DSR e podem ser utilizados na construção de sistemas de avaliação.

\section{Considerações Finais}

Alcançar o progresso em direção à sustentabilidade é claramente uma escolha da sociedade, das organizações, das comunidades e dos indivíduos. Como envolve diversas escolhas, a mudança apenas é possível se existir grande envolvimento da sociedade. Em resumo, o desenvolvimento sustentável força a sociedade a pensar em termos de longo prazo e a reconhecer o seu lugar dentro da biosfera. O conceito fornece uma nova perspectiva de observar o mundo e essa nova maneira tem mostrado que o estado atual da ativida- 
de humana é inadequado para preencher as necessidades vigentes. Além disso, está ameaçando seriamente a perspectiva de vida das futuras gerações.

Os objetivos do desenvolvimento sustentável desafiam as instituições contemporâneas que têm reagido às mudanças globais, relutando em reconhecer que esse processo esteja realmente ocorrendo. As diferenças em relação ao conceito de desenvolvimento sustentável são tão grandes que não existe um consenso sobre o que deve ser sustentado e tampouco sobre o que o termo sustentar significa. Consequentemente, não existe consenso sobre como medir a sustentabilidade. Infelizmente, para a maioria dos autores citados, sem uma definição operacional minimamente aceita torna-se impossível traçar estratégias e acompanhar o sentido e a direção do progresso.

Existe uma variedade de sistemas de indicadores que, atuando em diferentes dimensões, procuram mensurar a sustentabilidade do desenvolvimento. Cada um dos diferentes sistemas de avaliação apresenta características peculiares e é adequado para determinada realidade. Por outro lado, sistemas de indicadores adequados devem seguir alguns preceitos gerais importantes. A conformidade com tais preceitos, juntamente com a aplicação adequada da ferramenta para uma determinada realidade, está relacionada diretamente com o sucesso de um processo de avaliação.

Todas as definições e ferramentas relacionadas à sustentabilidade devem considerar o fato de que não se conhece totalmente como o sistema opera. Pode-se apenas descobrir os impactos ambientais decorrentes de atividades, e a interação com o bem-estar humano, com a economia e o meio ambiente. Em geral, se sabe que o sistema interage entre as diferentes dimensões, mas não se conhece especificamente o impacto dessas interações.

Os aspectos apresentados aqui mostram a diversidade e complexidade do termo desenvolvimento sustentável. Apesar da dificuldade que essas características conferem ao objeto de estudo, o desenvolvimento sustentável e a diversidade desse conceito devem servir não como obstáculo na procura de um melhor entendimento, mas, sim, como desafio na procura de novas visões acerca de ferramentas que procurem descrever a sustentabilidade. 


\section{Development Dimensions: a exploratory study under the perspective of the evaluation tools}

\section{Abstract}

This article explores the concept of development to focus afterwards in some tools of evaluation that work with this concept from different dimensions. In its first part the article discusses the different dimensions of development in order to present some tools that work with this concept. These tools are analyzed from several dimensions used in the evaluation process. One observes that the economic dimension is still predominant in the evaluation systems, however, significant advancements have been made in the development and application of evaluation systems that use the ecological and social dimension.

Key words: Sustainable Development. Indicators. Evaluation.

\section{Referências}

ANAND, Sudhir; SEN, Amartya. Sustainable Human Development: Concepts and Priorities. Occasional Paper \#8, Human Development Report Office, UNDP, New York, 1994.

BARTELMUS, P. Indicators of Sustainable Growth and Development Linkage Integration and Policy Use. Background Paper for Scientific Workshop on Indicators of Sustainable Development, Wuppertal, 15-17, november, 1995.

CAVAlCANTI, C. (Org.). Meio Ambiente, Desenvolvimento Sustentável e Políticas Públicas. São Paulo: Cortez, 1997.

COLEMAN, J. Human Capital in the Creation of Social Capital. In: American Journal of Sociology, v. 94: Supplement S101, 1988.

DAHL, Arthur L. The Big Picture: Comprehensive Approaches. In: MOLDAN, B.; BILHARZ, S. (Eds.) Sustainability Indicators: Report of the project on Indicators of Sustainable Development. Chichester: John Wiley \& Sons Ltd., 1997. 
DALY, H. Steady - State Economics: concepts, questions, policies. Gaia 1, p. 333-338, 1992.

. For the common good: redirecting the economy toward community, the environment, and a sustainable future. Boston: Beacon Press, 1994.

DALY, H. E.; COBB, J. For the Common Good: Redirecting the Economy Towards Community, the Environment and Sustainable Future. Boston: Beacon Press, 1989.

DASGUPTA, P. Human Well-Being and the Natural Environment.

Oxford: Oxford University Press, 2004.

GUIMARÃES, Roberto P. Desenvolvimento Sustentável: da retórica à formulação de políticas públicas. In: BECKER, B. K.; MIRANDA, M. (Orgs.). A Geografia Política do Desenvolvimento Sustentável. Rio de Janeiro: Editora UFRJ, 1997.

HAMMOND, A. et al. Environmental Indicators: A systematic approach to measuring and reporting on environmental policy performance in the context of sustainable development. Washington, DC.: World Resources Institut, 1995.

HARDI, P.; BARG, S. Measuring Sustainable Development: Review of Current Practice. Winnipeg: IISD,1997.

KANBUR, R. Economics, Social Science and Development. World Development, v. 30, n. 3, p. 477-486, 2002.

MACGILLIVRAY, A. Social Development Indicators. In: Sustainability Indicators: report of the project on indicators of sustainable development. Chichester: John Wiley \& Sons Ltd., 1997.

MACNEILL, J. et al. Beyond Interdependence. New York: Oxford University Press, 1991.

MCKINLEY, T. Linking Sustainability to Human Deprivations. In:

Sustainability Indicators: Report of the project on Indicators of Sustainable Development. Chichester: John Wiley \& Sons Ltd., 1997.

MEINE, C. Correction Lines: essays on land, leopold, and conservation. London, Island Press, 2004. 
MOLDAN, B; DAHL, A. L. Challenges to Sustainability Indicators. In: HAK, T.; MOLDAN, B.; DAHL, A. L. (Eds.) Sustainability Indicators: a scientific assessment. London: Island Press, 2007.

MOLDAN, B.; BILHARZ, S. (Eds.) Sustainability Indicators: report of the project on indicators of sustainable development. Chichester: John Wiley \& Sons Ltd., 1997.

MORSE, S. et al. Sustainability Indicators: The Problem of Integration.

Sustainable Development, n. 9, p. 1-15, 2001.

O'CONNOR, J. C. Measuring Wealth and Genuine Saving. In: Sustainability Indicators: report of the project on indicators of sustainable development. Chichester: John Wiley \& Sons Ltd., 1997.

OECD. Organization for Economic Cooperation and Development: core set of indicators for environmental performance reviews; a synthesis report by the group on the state of the environment. Paris: OECD, 1993.

PUTMAN, R. Bowling Alone: democracy in america at the end of the twentieth century. unpublished paper, Harward University, 1994.

RUTHERFORD, I. Use of Models to link Indicators of Sustainable Dvelopment. In: Moldan, B.; Bilharz, S. (Eds.) Sustainability Indicators: report of the project on indicators of sustainable development. Chichester: John Wiley \& Sons Ltd., 1997.

SACHS, I. Desenvolvimento Sustentável, Bio-Industrialização Descentralizada e Novas Configurações Rural-Urbanas. Os casos da Índia e do Brasil. In: VIEIRA, P. F.; WEBER, J. (Orgs.) Gestão de Recursos Naturais Renováveis e Desenvolvimento: novos desafios para a pesquisa ambiental. São Paulo: Cortez, 1997.

SAYER, J.; CAMPBELL, B. The Science of Sustainable Development: local livelihoods and the global environment. Cambridge: Cambridge University Press, 2004.

SEN, Amartya. The Standard of Living. Cambridge: University Press, 1987.

Desenvolvimento como Liberdade. São Paulo: Companhia das Letras, 2000. 
SERAGELDIN, Ismail. Sustainability and the Wealth of Nations: first steps in an ongoing journey. Enviromentally Sustainable Development Studies and Monograph Series n. 5. Washington, DC: The World Bank, 1996.

SERAGELDIN, I.; STEER, A. Epilogue: expanding the capital stock. In: SERAGLDIN, Ismail; STEER, Andrew (Eds.). Making Development Sustainable: from concepts to action. Enviromentally Sustainable Development Occasional Papers 2. Washington, DC: The World Bank, 1994.

STIGLITZ, Joseph E. A Globalização e seus Malefícios: a promessa não cumprida dos benefícios globais. São Paulo: Futura, 2002.

TURNER, R. K. et al. Environmental Economics: an elementary introduction. Baltimore: The John Hopkins University Press, 1993.

UNITED NATIONS DEVELOPMENT PROGRAMME (UNDP). Human Development Report. New York: Oxford University Press, 1990.

UNITED NATIONS DEVELOPMENT PROGRAMME (UNDP). Human Development Report. New York: Oxford University Press, 1995.

UNITED NATIONS (UN). Report of the United Nations Conference on Environment and Development, Rio de Janeiro, 2-14 June 1992, v. I, Resolutions Adopted by the Conference, Annex II, Agenda 21. A/CONF. 151/26/ REV. 1(v. I), p. 9-479, 1993.

UNITED NATIONS (UN). Work Programme on Indicators of Sustainable Development of the Commission on Sustainable Development.

Prepared by the Division For Sustainable Development in the Department for Policy Coordination and Sustainable Development, New York: United Nations, 1996a.

UNITED NATIONS (UN). Indicators of Sustainable Development: framework and methodologies. New York: United Nations, 1996b.

UNITED NATIONS (UN). Global change and sustainable development: critical trends. Economic and Social Council, Comission on Sustainable Development. E/CN. 17/1997/3, 1997.

WEIZSÄCKER, E. U. et al. Faktor vier. München: Drömer Knaur, 1995.

WORLD BANK (WB). Monitoring Environmental Progress: a report on work progress. Washington, DC.: World Bank, 1995. 